verfügbarkeit von MTX sein. Diese wiederum steht offenbar in Zusammenhang mit der Darmflora der Patienten, wie die vorliegende Studie zeigt.

Für diese Studie wurden an verschiedenen Kliniken in New York Stuhlproben von 47 bisher unbehandelten Patienten mit aktiver, neu aufgetretener RA analysiert (Trainingskohorte/26, Validierungskohorte/21). Die ersten Proben wurden 48 Stunden vor der ersten MTX-Gabe untersucht; weitere Proben folgten dann ein, zwei und vier Monate nach Therapiebeginn.

In der RNA-Sequenzierung der ersten 26 Proben zeigte sich, dass die Darmflora der Therapieresponder (Verbesserung des Krankheitsaktivitätsscores in 28 Gelenken $\geq 1,8$ vier Monate nach Therapiebeginn; 39\%) vor Therapiebeginn eine signifikant geringere mikrobielle Diversität aufwies als die der Nicht-Responder (61\%). Signifikante Differenzen gab es auch in der mikrobiellen Struktur. So waren vor Therapiebeginn in der Darmflora von Patienten, die nicht auf die Therapie ansprachen, beispielsweise größere Mengen der Stämme Firmicutes und Euryarchaeota, dafür aber weniger Bacteroidetes-Stämme nachzuweisen.

Da mit der RNA-Sequenzierung die metabolische Leistung der Bakterien nur annäherungsweise bestimmt werden kann, untersuchten die Autoren in der Trainingskohorte zusätzlich das Metagenom mittels Shotgun-Sequenzierung. Auch hier ergaben sich Unterschiede zwischen Respondern und Nicht-Respondern; bei letzteren

\section{Einfluss der Darmflora auf das Therapieansprechen bei MTX-Behandlung}

Artacho A, Isaac S, Nayak R et al. The Pretreatment Gut Microbiome Is Associated With Lack of Response to Methotrexat in New-Onset Rheumatoid Arthritis. Arthritis \& Rheumatology 2021; 73: 931-942

Methotrexat (MTX) ist zwar eins der wichtigsten Elemente der Behandlung der rheumatoiden Arthritis (RA), doch auf eine Monotherapie spricht nur die Hälfte der Patienten adäquat an. Ein möglicher Grund hierfür kann die interindividuell sehr unterschiedliche Biowaren z. B. 26 Signalwege signifikant erhöht (z. B. MAPK, DNA-Replikation, Fettsäureabbau) und 28 vermindert (z. B. Lipopolysaccharidsynthese, Kohlenhydratmetabolismus). Zudem ergaben sich Hinweise auf Veränderungen des bakteriellen Metabolismus von MTX, Folsäure und anderen Molekülen, die mit einem unzureichenden Ansprechen auf MTX in Verbindung stehen.

Die festgestellten Unterschiede im Metagenom bildeten die Grundlage für ein Mikrobiom-basiertes Model, mit dem das Therapieansprechen vorausgesagt werden kann. Mit den Daten aus der Trainingskohorte wurden 38 Metagenom-Merkmale ermittelt, die in das Modell eingebracht wurden. Viele dieser Merkmale haben bisher keinen bekannten Einfluss auf die Folsäure/MTXSignalwege, andere wiederum spielen beim Metabolismus von Thiamin eine Rolle, ein Nebenprodukt, dass den MTX-Transport stören kann. Die Testung des Modells in der Validierungskohorte zeigte, dass damit rund $80 \%$ der Patienten korrekt als Responder oder Non-Responder klassifiziert werden können. Allerdings scheint dies nur für die hier überprüfte Patientengruppe möglich zu sein. Denn das Modell wurde bei 20 weiteren Patienten getestet, die entweder unbehandelt waren oder andere antirheumatische Medikamente erhielten: Bei ihnen konnte das Therapieansprechen mit Hilfe des Modells nicht vorhergesagt werden. Und auch mit einem Modell, das auf den unterschiedlichen Bakterienstämmen beruhte, konnten die Patienten ebenfalls nicht sicher klassifiziert werden.

Um den Zusammenhang zwischen der Darmflora und dem Therapieansprechen zu ermitteln, analysierten die Autoren schließlich MTX und die bakteriell produzierten Metabolite in inkubierten Stuhlproben weiterer RA-Patienten, die mit MTX behandelt 
wurden. Das Mikrobiom einiger Patienten konnte die MTX-Konzentration sehr schnell reduzieren - diese Proben stammten überwiegend von Non-Respondern. In anderen Proben wiederum - hauptsächlich von Respondern - veränderte sich die Konzentration kaum.

\section{FAZIT}

Die Darmflora unterscheidet sich zwischen MTX-Respondern und Non-Respondern so stark, dass das prätherapeutische Mikrobiom für die Prädiktion des Therapieansprechens genutzt werden kann. Dabei ist allerdings weniger die dominante Spezies entscheidend, als vielmehr einzelne Bakteriengruppen und ihre metabolischen Funktionen. Ob die Darmflora MTX direkt verstoffwechseln kann oder ob sie das Therapieansprechen über das Immunsystem erhöht, sollte in weiteren Studien untersucht werden.

Stephanie Gräwert, Leipzig 